\title{
A Plane Symmetric 6R Foldable Ring
}

\author{
A.D. Viquerat ${ }^{\mathrm{a},}$, T. Hutt ${ }^{\mathrm{b}}$, S.D. Guest ${ }^{\mathrm{a}}$ \\ ${ }^{a}$ Department of Engineering, University of Cambridge, Trumpington Street, Cambridge CB2 1PZ, UK \\ ${ }^{b}$ Department of Mechanical Engineering, Imperial College London, Exhibition Road, South Kensington, London SW7 $2 A Z$, UK
}

\begin{abstract}
The design of a deployable structure which deploys from a compact bundle of six parallel bars to a rectangular ring is considered. The structure is a plane symmetric Bricard linkage. The internal mechanism is described in terms of its Denavit-Hartenberg parameters; the nature of its single degree of freedom is examined in detail by determining the exact structure of the system of equations governing its movement; a range of design parameters for building feasible mechanisms is determined numerically; and polynomial continuation is used to design rings with certain specified desirable properties.
\end{abstract}

Keywords: Numerical continuation, Deployable structure, Bricard linkage

\section{Introduction}

Several different types of foldable frames, which in their deployed configurations form (often regular) polygons, have appeared in literature in the past 40 years. Bennett or Bricard [1] linkages are frequently used as the basis linkages for foldable deployable frames. An early example appears in [2], in which an even number of bars are linked together in such a way that they can be folded into a tight bundle, and unfolded to form a regular polygon. In the six bar case, a three-fold symmetric linkage results [3], while in the four bar case, a Bennett linkage is formed [4, 5]. Four bar foldable frames have been extensively examined $[6,4,7]$. For the six-bar case, Wohlhart [8,9] and Racilla [10] have focussed on the kinematics of the trihedral, or 'rectangular' member of the Bricard family. In [11], Pellegrino et al. proposed a new family of six bar foldable frames. A two-fold symmetric member of this family has been proposed as a support for a solar blanket, and its kinematics examined numerically $[12,13]$. Recently, the two-fold symmetric 6R foldable frame was identified as a special line and plane symmetric Bricard linkage [14]. This particular variant does, however, suffer from problems with bifurcations (although certain designs avoid this). If one of the two planes of symmetry is removed, a mobile $6 \mathrm{R}$ ring which experiences fewer problems with bifurcations remains. An example is shown in Figure 1. In this paper, a greater understanding of the plane symmetric $6 \mathrm{R}$ foldable ring is sought by first identifying the ring as a plane symmetric Bricard linkage, examining the nature of its mobility using a cascade of homotopies [15] to identify positive dimensional solution sets (an application of polynomial continuation), determining a range of design parameters for building feasible mechanisms of this type, deriving a closed form expression for the linkage's kinematics, and finally employing polynomial continuation, again, in an attempt to design a family of plane symmetric $6 \mathrm{R}$ foldable rings with certain desirable practical properties.

\section{Linkage Specification}

The two-fold symmetric ring of [14] has two different bar lengths $\left(l_{1}\right.$ and $\left.l_{2}\right)$, and the bars are all tilted from the vertical by a single angle $\mu$. Square cross-sectioned, prismatic (i.e., untwisted) bars were used. By contrast, the 6R linkage considered here has all six bar lengths the same (l), and four separate bar tilt angles $\left(\alpha_{1}^{\prime}, \alpha_{2}^{\prime}, \beta_{1}\right.$ and $\left.\beta_{2}\right)$, introducing a requirement that, if the bars have a square cross-section, some of the bars must be twisted in order to

Email address: sdg@eng. cam.ac.uk (S.D. Guest) 
match the prescribed tilt angles at each end. A simplified diagram of the deployed linkage with all design parameters labelled is given in Figure 3, while a representation of the physical linkage is given in Figure 4, in which the twists in the bars are clearly visible. While a square cross-section is not required to construct the linkage, it does aid in visualising the bar twist.

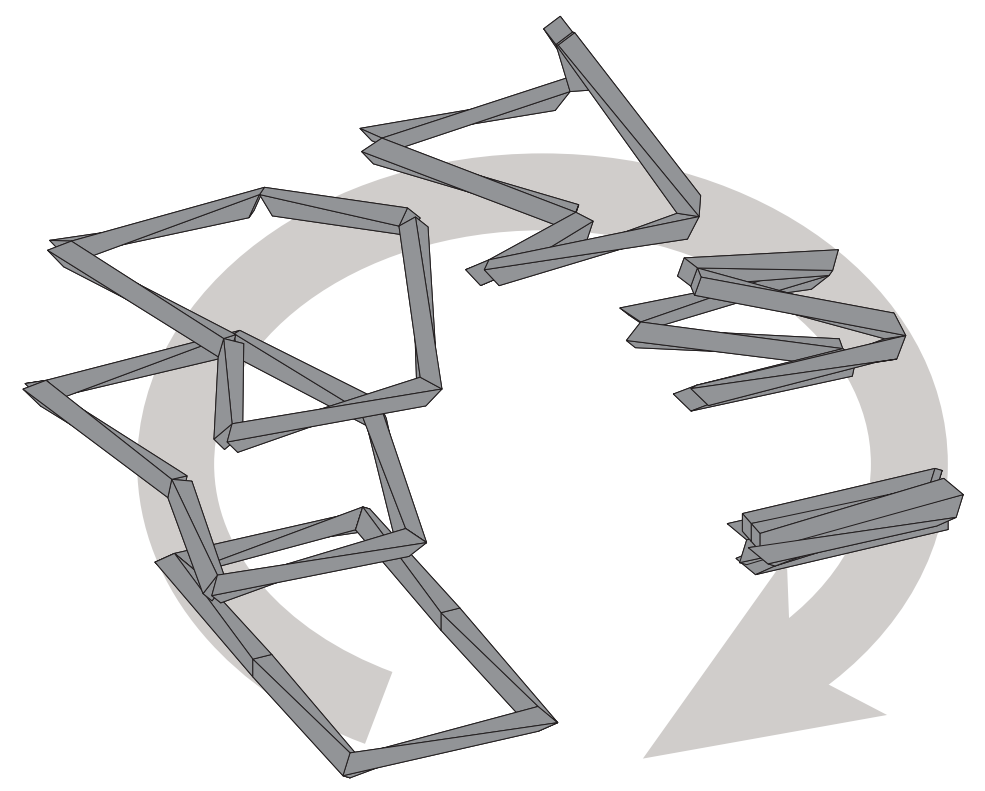

Figure 1: Folding process for a linkage with parameters $\alpha_{1}=\pi / 4, \alpha_{2}=-\pi / 4$ and $\gamma=\pi / 2$. Each bar is shown as a twisted prismatic bar with square cross-section.
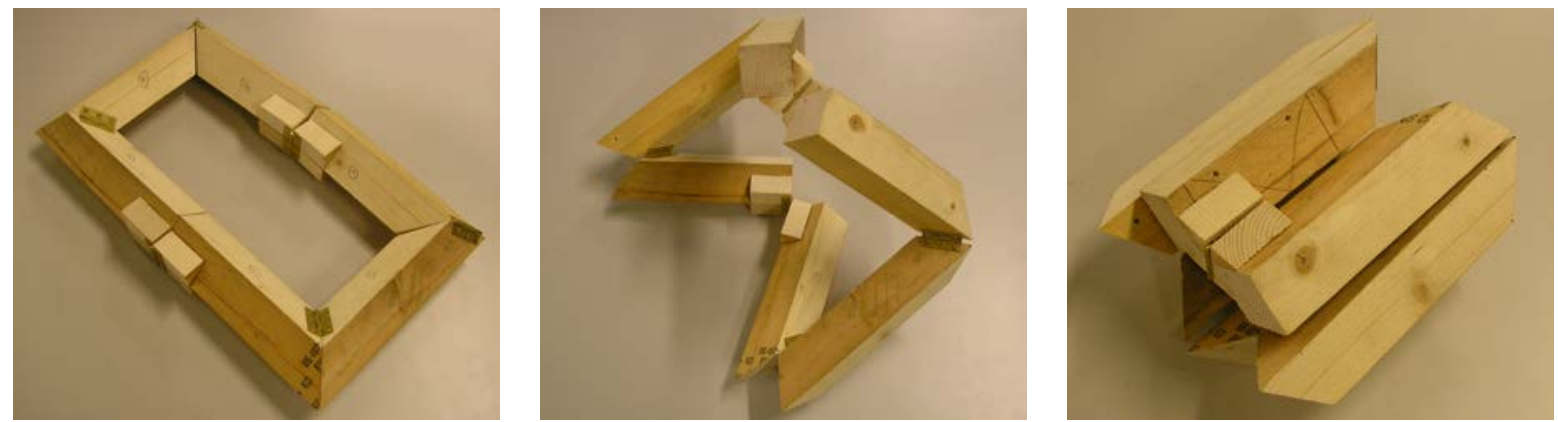

Figure 2: The folding of a wooden model of the linkage shown in Figure 1.

There are six hinges (labelled $\mathbf{h}_{1}-\mathbf{h}_{6}$ ), each with a single rotational degree of freedom connecting the bars in a closed loop. The plane of symmetry is preserved through the folding motion. It is labelled as the $X Z$ plane in the fully deployed/open configuration, shown in Figure 3. The plane contains the points $\mathbf{p}_{6}$ and $\mathbf{p}_{3}$, and the vectors $\mathbf{h}_{6}$ and $\mathbf{h}_{3}$, which are inclined to the $Z$ axis by angles $\beta_{1}$ and $\beta_{2}$ respectively. Also when deployed, hinges $\mathbf{h}_{1}$ and $\mathbf{h}_{2}$ lie in planes rotated from the $Y Z$ plane by $45^{\circ}$ about the $Z$ axis. The angles these hinges form to the horizontal can be specified in two important ways. When constructing physical models of the plane-symmetric 6-bar, the most intuitive form is obtained by taking the projection of the hinges onto the $Y Z$ plane, and considering the angle formed between that projection and the $X Y$ plane, labelled here as $\alpha_{1}^{\prime}$ and $\alpha_{2}^{\prime}$. This projection is shown in Figure 3. This is a more intuitive 


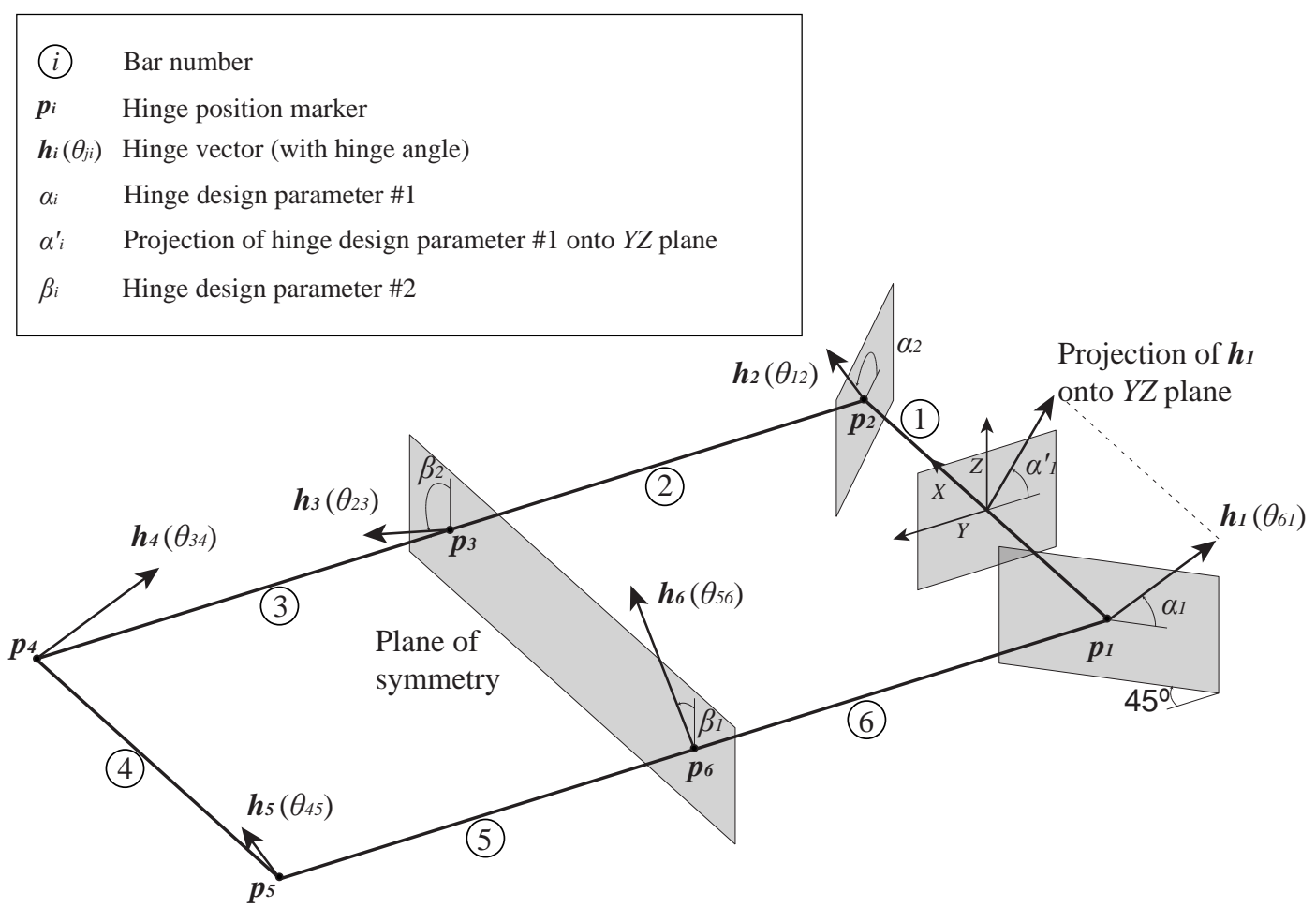

Figure 3: Design variables of the plane-symmetric 6R linkage with plane of symmetry shown.

definition as it specifies the tilt angle that a square cross-sectioned bar would need to form with the horizontal before cuts at $45^{\circ}$ are made. However, future mathematical results are simplified by directly taking the angle between the $X Y$ plane and the hinge vectors, written as $\alpha_{1}$ and $\alpha_{2}$ here. Relationships between the two $\alpha$ definitions can be constructed as:

$$
\begin{aligned}
\tan \alpha & =\frac{1}{\sqrt{2}} \tan \alpha^{\prime} \\
\Rightarrow \sin \alpha & =\frac{\sin \alpha^{\prime}}{\sqrt{\sin ^{2} \alpha^{\prime}+2 \cos ^{2} \alpha^{\prime}}} \\
\Rightarrow \sin ^{2} \alpha & =\frac{\sin ^{2} \alpha^{\prime}}{1+\cos ^{2} \alpha^{\prime}} \\
\cos ^{2} \alpha & =\frac{2 \cos ^{2} \alpha^{\prime}}{1+\cos ^{2} \alpha^{\prime}}
\end{aligned}
$$

In order for all the bars to be parallel when fully stowed, the hinge vectors $\mathbf{h}_{6}$ and $\mathbf{h}_{3}$ must also be parallel in the fully folded configuration since they must be perpendicular to both the plane of symmetry and the ends of the bars to which they are attached. In general, $\mathbf{h}_{6}$ and $\mathbf{h}_{3}$ will not be parallel when folded for a given choice of $\beta_{1}$ and $\beta_{2}$. The parallel condition can be enforced by specifying a simple linear relationship:

$$
\begin{aligned}
& \beta_{1}=2 \alpha_{1}^{\prime}-\gamma \\
& \beta_{2}=\pi-2 \alpha_{2}^{\prime}+\gamma
\end{aligned}
$$

where an extra variable, $\gamma$, has been introduced to replace $\beta_{1}$ and $\beta_{2}$. The variable $\gamma$ describes the relative rotation of the two end bars in the folded state. Note that it is the (projected) $\alpha_{1}^{\prime}$ and $\alpha_{2}^{\prime}$ definitions which have been used here. Three variables $\left(\left\{\alpha_{1}^{\prime}, \alpha_{2}^{\prime}, \gamma\right\}\right.$ or equivalently $\left.\left\{\alpha_{1}, \alpha_{2}, \gamma\right\}\right)$ remain to specify the linkage. 


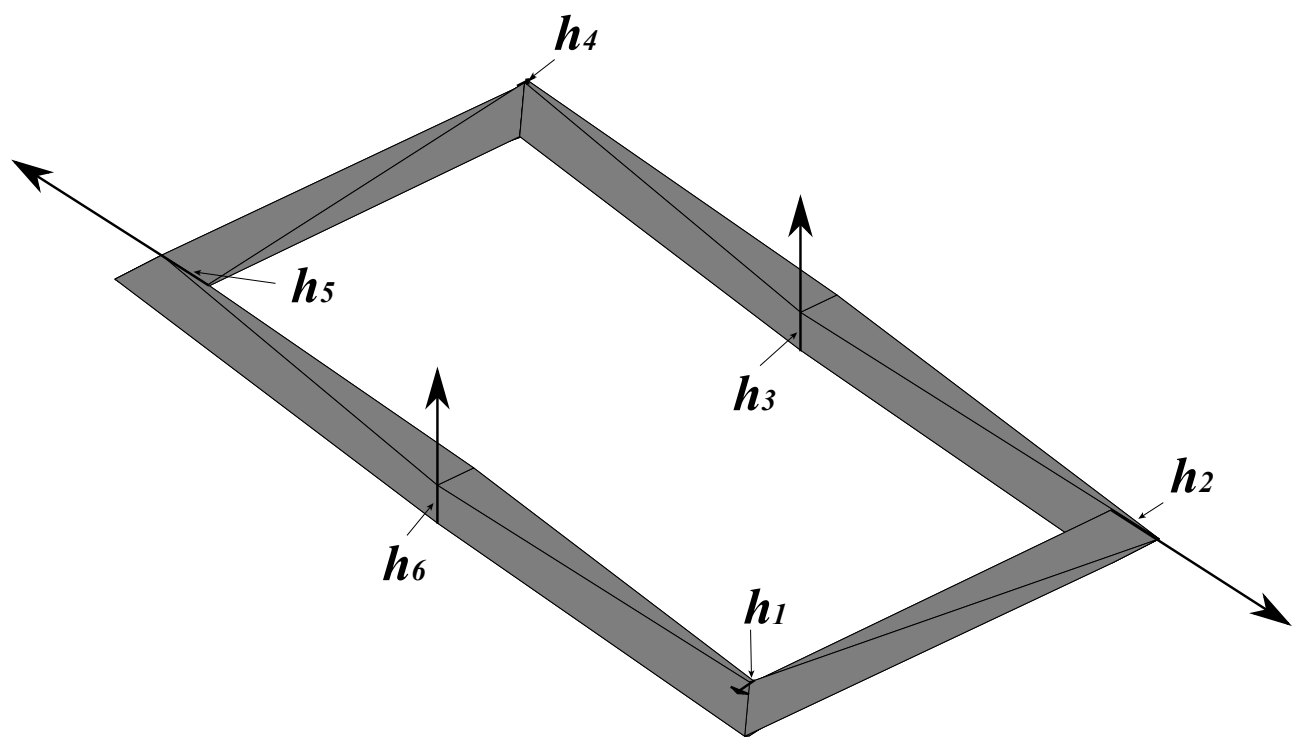

Figure 4: Example of plane-symmetric 6R foldable linkage with visible bar twist for $\alpha_{1}=\pi / 4, \alpha_{2}=-\pi / 4$ and $\gamma=\pi / 2$.

It can be shown that hinges 1 and 2 have maximum opening angles (assuming $\theta_{i j}=0$ when fully deployed):

$$
\begin{aligned}
& \theta_{61 \text { max }}=\pi-2 \tan ^{-1}\left[\frac{\tan \alpha_{1}^{\prime}}{\sqrt{2+\tan ^{2} \alpha_{1}^{\prime}}}\right]=\pi-\cos ^{-1}\left(\cos ^{2} \alpha_{1}^{\prime}\right) \\
& \theta_{12 \max }=\pi-2 \tan ^{-1}\left[\frac{\tan \alpha_{2}^{\prime}}{\sqrt{2+\tan ^{2} \alpha_{2}^{\prime}}}\right]=\pi-\cos ^{-1}\left(\cos ^{2} \alpha_{2}^{\prime}\right)
\end{aligned}
$$

At these maximum angles, bars 6 and 2 are both colinear with bar 1, indicating full folding of the linkage. These definitions will be useful for simulation purposes later.

\section{Identification as a Bricard Plane Symmetric Case}

Figure 5 illustrates the Denavit-Hartenberg parameters for the plane symmetric Bricard linkage. The relationships (implied by symmetry) between these parameters (from [16]) are:

$$
\begin{aligned}
& a_{61}=a_{56}, a_{12}=a_{45}, a_{23}=a_{34} \\
& \alpha_{61}+\alpha_{56}=\alpha_{12}+\alpha_{45}=\alpha_{23}+\alpha_{34}=\pi \\
& R_{6}=R_{3}=0, R_{1}=R_{5}, R_{2}=R_{4}
\end{aligned}
$$

It is possible to derive a relationship between the design variables $\alpha_{1}, \alpha_{2}, \beta_{1}$ and $\beta_{2}$ of the plane symmetric $6 \mathrm{R}$ foldable ring, and the Denavit-Hartenberg parameters. This is achieved by solving a series of simple linear equations which arise from the linkage's geometry. Start with the link between hinges 6 and 1. The Bricard linkage joints must 


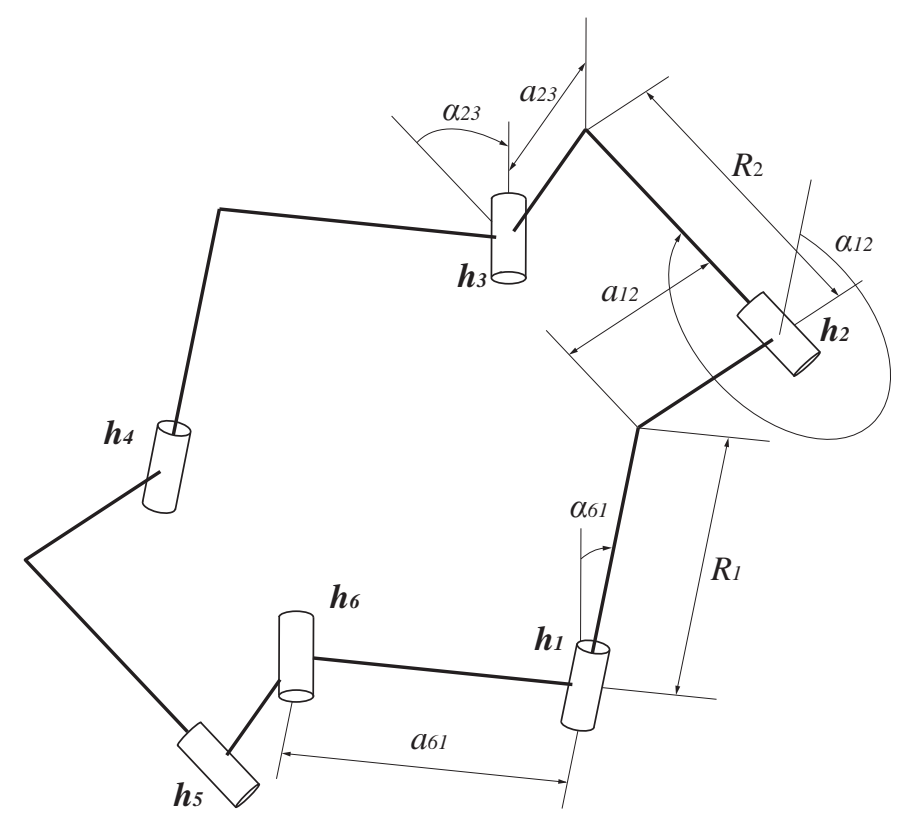

Figure 5: Plane symmetric Bricard linkage (case illustrated is from linkage with $\alpha_{1}=\pi / 4, \alpha_{2}=-\pi / 4$ and $\gamma=\pi / 2$ ). The plane of symmetry is defined by hinges $\mathbf{h}_{6}$ and $\mathbf{h}_{3}$.

lie on a line which passes through $\mathbf{p}_{i}(0)$ and is parallel to $\hat{\mathbf{h}}_{i}(0)$, where the notation $(0)$ represents positions and vectors in the deployed configuration, and $\hat{\mathbf{h}}$ represents a unit vector:

$$
\begin{aligned}
& \mathbf{p}_{6}=\mathbf{p}_{6}(0)+t_{l 6} \hat{\mathbf{h}}_{6}(0) \\
& \mathbf{p}_{1}=\mathbf{p}_{1}(0)+t_{l 1} \hat{\mathbf{h}}_{1}(0)
\end{aligned}
$$

Here, $t_{l 6}$ and $t_{l 1}$ are unknown scaling factors. It is apparent that $R_{6}=0$, but also that $a_{61}$ is non-zero. $a_{61}$ is perpendicular to both $\hat{\mathbf{h}}_{6}(0)$ and $\hat{\mathbf{h}}_{1}(0)$, so define a new vector of unit length as:

$$
\mathbf{a}_{61}^{\prime}=\hat{\mathbf{h}}_{6}(0) \times \hat{\mathbf{h}}_{1}(0)
$$

The length of $a_{61}$ is unknown, so a third scaling factor, $t_{a 61}$, is introduced. The governing equation is given in Equation 4.

$$
\begin{array}{rlrl} 
& \mathbf{p}_{6}+t_{a 61} \mathbf{a}_{61}^{\prime} & =\mathbf{p}_{1} \\
\Rightarrow \mathbf{p}_{6}(0)+t_{l 6} \hat{\mathbf{h}}_{6}(0)+t_{a 61} \mathbf{a}_{61}^{\prime} & =\mathbf{p}_{1}(0)+t_{l 1} \hat{\mathbf{h}}_{1}(0)
\end{array}
$$

This can easily be solved to give $t_{l 6}, t_{11}$ and $t_{a 61}$, and hence $\mathbf{p}_{6}, \mathbf{p}_{1}$ and $a_{61}=\left|t_{a 61} \mathbf{a}_{61}^{\prime}\right|$.

Moving on to the next link (between hinges 1 and 2), define:

$$
\mathbf{p}_{2}=\mathbf{p}_{2}(0)+t_{l 2} \hat{\mathbf{h}}_{2}(0)
$$

as well as:

$$
\mathbf{a}_{12}^{\prime}=\hat{\mathbf{h}}_{1}(0) \times \hat{\mathbf{h}}_{2}(0)
$$

Since $R_{1}$ is non-zero, introduce the unknown $t_{R 1}$ to the governing Equation 5 .

$$
\begin{aligned}
\mathbf{p}_{1}+t_{R 1} \hat{\mathbf{h}}_{1}(0) & =\mathbf{p}_{2}+t_{a 12} \mathbf{a}_{12}^{\prime} \\
\Rightarrow \mathbf{p}_{1}+t_{R 1} \hat{\mathbf{h}}_{1}(0) & =\mathbf{p}_{2}(0)+t_{l 2} \hat{\mathbf{h}}_{2}(0)+t_{a 12} \mathbf{a}_{12}^{\prime}
\end{aligned}
$$


From this $t_{l 2}, t_{R 1}$ and $t_{a 12}$ can be found, which in turn gives $\mathbf{p}_{2}, R_{1}=t_{R 1}$ and $a_{12}=\left|t_{a 12} \mathbf{a}_{12}^{\prime}\right|$.

Finally, consider the link between hinges 2 and 3 by defining:

$$
\mathbf{p}_{3}=\mathbf{p}_{3}(0)+t_{l 3} \hat{\mathbf{h}}_{3}(0)
$$

as well as:

$$
\mathbf{a}_{23}^{\prime}=\hat{\mathbf{h}}_{2}(0) \times \hat{\mathbf{h}}_{3}(0)
$$

and Equation 6.

$$
\begin{aligned}
\mathbf{p}_{2}+t_{R 2} \hat{\mathbf{h}}_{2}(0) & =\mathbf{p}_{3}+t_{a 23} \mathbf{a}_{23}^{\prime} \\
\Rightarrow \mathbf{p}_{2}+t_{R 2} \hat{\mathbf{h}}_{2}(0) & =\mathbf{p}_{3}(0)+t_{l 3} \hat{\mathbf{h}}_{3}(0)+t_{a 23} \mathbf{a}_{23}^{\prime}
\end{aligned}
$$

From this $t_{l 3}, t_{R 2}$ and $t_{a 23}$ can be found, which in turn gives $\mathbf{p}_{3}, R_{2}=t_{R 2}$ and $a_{23}=\left|t_{a 23} \mathbf{a}_{23}^{\prime}\right|$.

The important parameters (obtained using Mathematica [17]) are:

$$
\begin{aligned}
& \frac{a_{61}}{l}=\sqrt{1-\frac{4 \cos ^{2}\left(\alpha_{1}\right)}{2 \sqrt{2} \sin \left(2 \alpha_{1}\right) \sin \left(2 \beta_{1}\right)+\left(3 \cos \left(2 \alpha_{1}\right)-1\right) \cos \left(2 \beta_{1}\right)+\cos \left(2 \alpha_{1}\right)+5}} \\
& \frac{a_{12}}{l}=2 \sqrt{\frac{\sin ^{2}\left(\alpha_{1}-\alpha_{2}\right)}{2 \cos \left(2 \alpha_{1}\right)+4 \sin ^{2}\left(\alpha_{1}\right) \cos \left(2 \alpha_{2}\right)+6}} \\
& \frac{a_{23}}{l}=\sqrt{1-\frac{4 \cos ^{2}\left(\alpha_{2}\right)}{-2 \sqrt{2} \sin \left(2 \alpha_{2}\right) \sin \left(2 \beta_{2}\right)+\left(3 \cos \left(2 \alpha_{2}\right)-1\right) \cos \left(2 \beta_{2}\right)+\cos \left(2 \alpha_{2}\right)+5}}
\end{aligned}
$$

and

$$
\begin{aligned}
& \frac{R_{1}}{l}=\sqrt{2}\left(\frac{4 \cos \left(\alpha_{1}\right)}{2 \sqrt{2} \sin \left(2 \alpha_{1}\right) \sin \left(2 \beta_{1}\right)+\left(3 \cos \left(2 \alpha_{1}\right)-1\right) \cos \left(2 \beta_{1}\right)+\cos \left(2 \alpha_{1}\right)+5}-\right. \\
& \left.\frac{2\left(\cos \left(\alpha_{1}\right)+\sin \left(\alpha_{1}\right) \sin \left(\alpha_{2}\right) \cos \left(\alpha_{2}\right)\right)}{\cos \left(2 \alpha_{1}\right)+2 \sin ^{2}\left(\alpha_{1}\right) \cos \left(2 \alpha_{2}\right)+3}\right) \\
& \frac{R_{2}}{l}=\sqrt{2} \sec \left(\alpha_{2}\right) \cdot\left(-\frac{4 \cos ^{2}\left(\alpha_{2}\right)}{-2 \sqrt{2} \sin \left(2 \alpha_{2}\right) \sin \left(2 \beta_{2}\right)+\frac{\left(3 \cos \left(2 \alpha_{2}\right)-1\right) \cos \left(2 \beta_{2}\right)+\cos \left(2 \alpha_{2}\right)+5}{2}+}\right. \\
& \left.\frac{\sin \left(2 \alpha_{1}\right) \sin \left(2 \alpha_{2}\right)+4 \cos \left(2 \alpha_{2}\right)+4 \sin ^{2}\left(\alpha_{2}\right) \cos \left(2 \alpha_{1}\right)+8}{2 \cos \left(2 \alpha_{1}\right)+4 \sin ^{2}\left(\alpha_{1}\right) \cos \left(2 \alpha_{2}\right)+6}-1\right)
\end{aligned}
$$

The twist angles can be simply determined as:

$$
\begin{aligned}
& \alpha_{61}=\cos ^{-1}\left(\left\langle\hat{\mathbf{h}}_{6}(0), \hat{\mathbf{h}}_{1}(0)\right\rangle\right) \\
& \alpha_{12}=\cos ^{-1}\left(\left\langle\hat{\mathbf{h}}_{1}(0), \hat{\mathbf{h}}_{2}(0)\right\rangle\right) \\
& \alpha_{23}=\cos ^{-1}\left(\left\langle\hat{\mathbf{h}}_{2}(0), \hat{\mathbf{h}}_{3}(0)\right\rangle\right)
\end{aligned}
$$

As a numerical example, consider the case of $\alpha_{1}=\pi / 4, \alpha_{2}=-\pi / 4$, and $\beta_{1}=\beta_{2}=0(\gamma=\pi / 2)$. This can be written in terms of Denavit-Hartenberg parameters for the plane symmetric case:

$$
\begin{aligned}
\frac{a_{61}}{l}=\frac{1}{\sqrt{2}}, \frac{a_{12}}{l} & =\sqrt{\frac{2}{3}}, \frac{a_{23}}{l}=\frac{1}{\sqrt{2}} \\
\frac{R_{1}}{l} & =\frac{2}{3}, \frac{R_{2}}{l}=-\frac{2}{3} \\
\alpha_{61}=\frac{\pi}{4}, \alpha_{12} & =\frac{2 \pi}{3}, \alpha_{23}=\frac{3 \pi}{4}
\end{aligned}
$$




\section{Loop Closure Equations}

It is possible to construct a set of loop closure equations by defining coordinate systems attached to each link, and then deriving the transfer matrices which describe the transformation from one coordinate system to the next. This method is illustrated in [14] and [13], where it is used to simulate the motion of closed loop linkages, and to study their bifurcations. A transfer matrix is typically $4 \times 4$, and consists of a $3 \times 3$ rotation matrix, say $R$, and a $3 \times 1$ translation vector, say $\mathbf{v}$. These parts are arranged as:

$$
T=\left[\begin{array}{cccc} 
& R & & \mathbf{v} \\
0 & 0 & 0 & 1
\end{array}\right]
$$

If a coordinate system is attached to the end of a link in a linkage, then a transfer matrix can be used to rotate and translate it to the location of the coordinate system attached to an adjacent link in a single operation. Repeating this operation around a linkage which is also a closed loop will eventually lead back to the original link. Mathematically, this can be expressed as:

$$
F=T_{61} T_{56} T_{45} T_{34} T_{23} T_{12}-I=0
$$

where $T_{a b}$ defines the transfer between the coordinate system attached to link $a$ to that attached to $b$. The coordinate system at each joint is aligned so that the $z$-axis is aligned with the hinge axis. Before each translation, the $x$-axis is rotated such that it points along the current bar towards the next joint. As there are single degree of freedom connections between the links, each transfer matrix can be separated into a part which deals only with rotation about the z-axis, $L_{3}$, and a part which relates to the unchanging geometry of the link, $T_{a b}^{L}$, and $T_{a b}=T_{a b}^{L} L_{3}\left(\theta_{a b}\right)$. The equations above then become:

$$
F=T_{61}^{L} L_{3}\left(\theta_{61}\right) T_{56}^{L} L_{3}\left(\theta_{56}\right) T_{45}^{L} L_{3}\left(\theta_{45}\right) T_{34}^{L} L_{3}\left(\theta_{34}\right) T_{23}^{L} L_{3}\left(\theta_{23}\right) T_{12}^{L} L_{3}\left(\theta_{12}\right)-I=0
$$

Explicitly, the transfer matrices for each of the six links are:

$$
\begin{aligned}
& T_{12}^{L}=L_{1}\left(\beta_{2}\right) M L_{3}(\pi / 4) L_{1}\left(\alpha_{2}-\pi / 2\right) \\
& T_{23}^{L}=L_{1}\left(\pi / 2-\alpha_{2}\right) L_{3}(\pi / 4) M L_{1}\left(-\beta_{2}\right) \\
& T_{34}^{L}=L_{1}\left(\pi / 2-\alpha_{1}\right) L_{3}(\pi / 4) M L_{3}(\pi / 4) L_{1}\left(\alpha_{2}-\pi / 2\right) \\
& T_{45}^{L}=L_{1}\left(-\beta_{1}\right) M L_{3}(\pi / 4) L_{1}\left(\alpha_{1}-\pi / 2\right) \\
& T_{56}^{L}=L_{1}\left(\pi / 2-\alpha_{1}\right) L_{3}(\pi / 4) M(\pi / 4) L_{1}\left(\beta_{1}\right) \\
& T_{61}^{L}=L_{1}\left(\pi / 2-\alpha_{2}\right) L_{3}(\pi / 4) M L_{3}(\pi / 4) L_{1}\left(\alpha_{1}-\pi / 2\right)
\end{aligned}
$$

where $L_{1}$ is a rotation about the $x$-axis, and $M$ defines a translation of length $l$ along each link:

$$
M=\left[\begin{array}{cccc}
1 & 0 & 0 & -l \\
0 & 1 & 0 & 0 \\
0 & 0 & 1 & 0 \\
0 & 0 & 0 & 1
\end{array}\right]
$$

The single off-diagonal entry in matrix $M$ has a negative sign because the effect of applying $M$ to a point in space is to rewrite that point as a location in the basis of the new, translated coordinate system. Shifting a coordinate system in the positive $x$-direction requires the inclusion of a negative term in the $(1,4)$ location of matrix $M$. The matrix Equation 7 can be separated into six individual equations which together ensure loop closure. As in [13], the strictly upper triangular part of this matrix equation provides six independent scalar equations necessary to form a square system:

$$
\mathbf{F}\left(\theta_{12}, \theta_{23}, \theta_{34}, \theta_{45}, \theta_{56}, \theta_{61}\right)=\left\{\begin{array}{l}
F_{1,2} \\
F_{1,3} \\
F_{1,4} \\
F_{2,3} \\
F_{2,4} \\
F_{3,4}
\end{array}\right\}=\mathbf{0}
$$


Having derived these loop closure equations, it is possible to simulate the motion of the linkage using a type of predictor-corrector approach detailed in [13] and [14]. One particularly useful by-product of this method is a matrix whose singular values can be used to examine the linkage's mobility at each point of the unfolding process. The structure of these equations will be examined in the following section.

\section{Examination of Mobility using a Cascade of Homotopies}

The plane-symmetric six-bar ring can be described as an over-constrained mechanism, or as possessing a geometric degree of freedom. This implies that it does not satisfy the Kutzbach Criterion [18], which must be due to some special feature of the linkage's geometry, in this case its symmetry. It is possible to determine mathematically if a linkage is likely to possess a geometric degree of freedom, and if so, of what order, and in how many disconnected sets, by using the method of witness sets described in [19] and [15]. This type of analysis forms part of a broader field known as Polynomial Continuation [20, 21, 22] in which all finite solutions to a system of polynomial equations are found by first finding the solutions to a separate system of equations with a similar polynomial structure, and then numerically tracking these known solutions into those of the unknown system. The method can be used to identify finite, geometrically isolated solutions, or with a few additions, to find curves of solutions through the solution space.

The loop closure equation of section 4 can be simplified by assuming that hinge angles reflected in the plane of symmetry will be equal, as:

$$
\begin{aligned}
& \theta_{12}=\theta_{34} \\
& \theta_{45}=\theta_{61}
\end{aligned}
$$

Recall that these hinge angles are defined as being zero when the foldable ring is in the fully deployed state. Finally, the maximum order of the resulting polynomial closure equations can be reduced by rearrangement into the form of Equation 10.

$$
\begin{aligned}
F^{\prime}= & L_{3}\left(\theta_{61}\right) T_{34}^{L} L_{3}\left(\theta_{12}\right) T_{23}^{L} L_{3}\left(\theta_{12}\right) T_{12}^{L}-\ldots \\
& \left(T_{45}^{L}\right)^{-1} L_{3}\left(-\theta_{56}\right)\left(T_{56}^{L}\right)^{-1} L_{3}\left(-\theta_{61}\right)\left(T_{61}^{L}\right)^{-1} L_{3}\left(-\theta_{12}\right)=\mathbf{0}
\end{aligned}
$$

which is written in terms of the four unknowns $\left\{\theta_{12}, \theta_{23}, \theta_{56}, \theta_{61}\right\}$. Equation 10 can be written in pure polynomial form by replacing the trigonometric functions of the four unknowns with new variables by setting $\cos \left(\theta_{i j}\right)=C_{i j}$ and $\sin \left(\theta_{i j}\right)=S_{i j}$. Four elements of Equation 10 can be chosen, and augmented with standard trigonometric identities relating the new variables, to construct a system of equations of the form:

$$
\left.\begin{array}{l}
C_{12}^{2}+S_{12}^{2}-1 \\
C_{23}^{2}+S_{23}^{2}-1 \\
C_{56}^{2}+S_{56}^{2}-1 \\
C_{61}^{2}+S_{61}^{2}-1 \\
F_{1,2}^{\prime} \\
F_{1,3}^{\prime} \\
F_{2,3}^{\prime} \\
F_{3,4}^{\prime}
\end{array}\right\}=\mathbf{0}
$$

Equation 11 defines a relationship between each of the hinge angles. It is a system of polynomial equations in which the coefficients are written in terms of the 6-bar design parameters $\alpha_{1}, \alpha_{2}, \beta_{1}, \beta_{2}$ and the bar length $l$. It has a mixed volume [23] of 176, which means there is a tight upper bound of 176 on the number of finite solutions. If this is, in fact, an over-constrained mechanism, then it can be expected that positive dimensional solution sets will be present. A positive dimensional solution set is a continuum of solutions which may exist on a line, plane, or higher dimensional manifold. Often, more than one curve or manifold of solutions will be present in a system of equations. If these curves do not intersect at any point, they are known as irreducible components. The appearance of positive dimensional solution sets in the closure equations of a linkage is a sign that the linkage may actually be mobile. The dimensionality of the solution set corresponds to the degree of freedom of the linkage. For example, if a one-dimensional solution set is found, then it is possible that the linkage will be mobile with a single degree 
of freedom. Since Equation 11 contains four unknowns, it is possible that there could be as high as a $n-1=3$ dimensional solution set. It is known a priori, however, that no three or two-dimensional solution sets are present. By introducing to the system an additional affine equation of dimension equal to that of the solution set sought and with randomly generated complex coefficients (a complex hyperplane), a witness set can be generated. After introducing a single, random complex intersecting hyperplane into the equations (increasing the mixed volume to 400), and solving the resulting system using standard continuation methods, it was discovered that Equation 11 has a one-dimensional solution set whose witness set contains 28 elements. This first step also generated a further 246 solutions which do not lie on a one-dimensional curve of solutions. To determine which of these extra solutions are actually zerodimensional geometrically isolated points, a cascade step is required [15]. The cascade involves gradually removing the intersecting plane, introduced earlier, from the equations by way of a new homotopy. Using this method a zerodimensional solution set, also with 28 members, was found. The members of the witness set were all found to belong to the same irreducible component which must, therefore, represent the single mobile path known to exist in the real linkage. The cascade process is represented in Figure 6.

It has been shown that the plane-symmetric six-bar linkage has a single irreducible component in one dimension, which has 28 members when the closure equations are posed as above. Since it is known that this linkage has a single geometric degree of freedom, it can be stated that this irreducible component of degree 28 is responsible for its mobility. The fact that a linkage's closure equations contain a positive dimensional solution set does not, in general, prove that a linkage will actually possess any mobility, but suggests that it may be possible. To prove that a linkage does have a degree of freedom would require showing that there is at least one irreducible component in purely real space, and that at least part of this lies in a feasible region of the linkage's parameters.

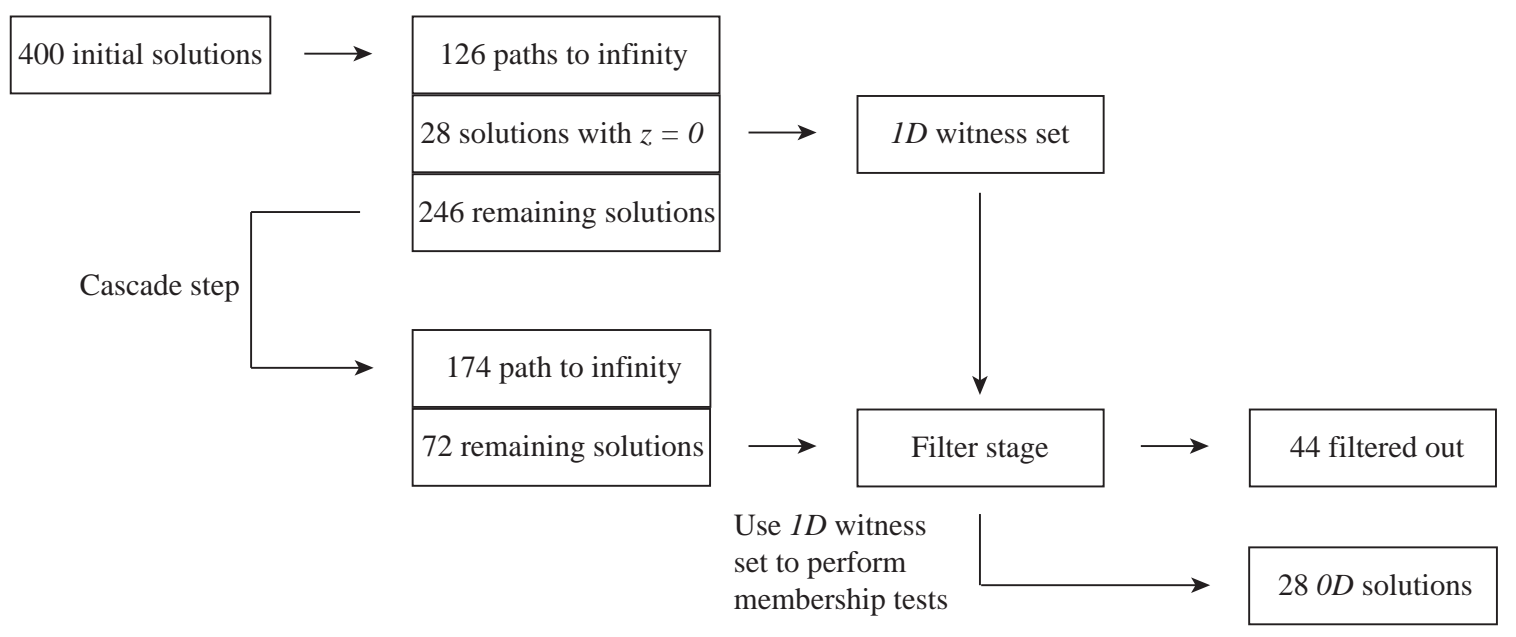

Figure 6: Homotopy cascade used to determine 1D and OD witness sets for 6R linkage.

\section{Finding Feasible Designs}

Only certain combinations of the three design parameters $\left\{\alpha_{1}, \alpha_{2}, \gamma\right\}$ will lead to a linkage which behaves in a way which is likely to be desirable. The most fundamental requirements of a linkage of the type under consideration are:

- linkage does not bifurcate at any point;

- linkage unfolds continuously and smoothly from closed to open (folded to deployed) configuration; 
- hinge angles are allowed only to lie in the range spanned by the same hinge's angle when deployed, and the angle when stowed;

- no two bars intersect during the unfolding process.

It has been observed that the satisfaction of the first three points ensures the satisfaction of the fourth, and so this fourth point is not considered here.

Since there are only three design variables, it is possible to hold one of them constant, and construct a 2dimensional plot depicting the feasible space in terms of the other two. Holding $\gamma$ constant, a set of feasibility contour lines can be plotted, as in Figure 7. The $\gamma$ dependence of the feasible region is indicated in Figure 8.
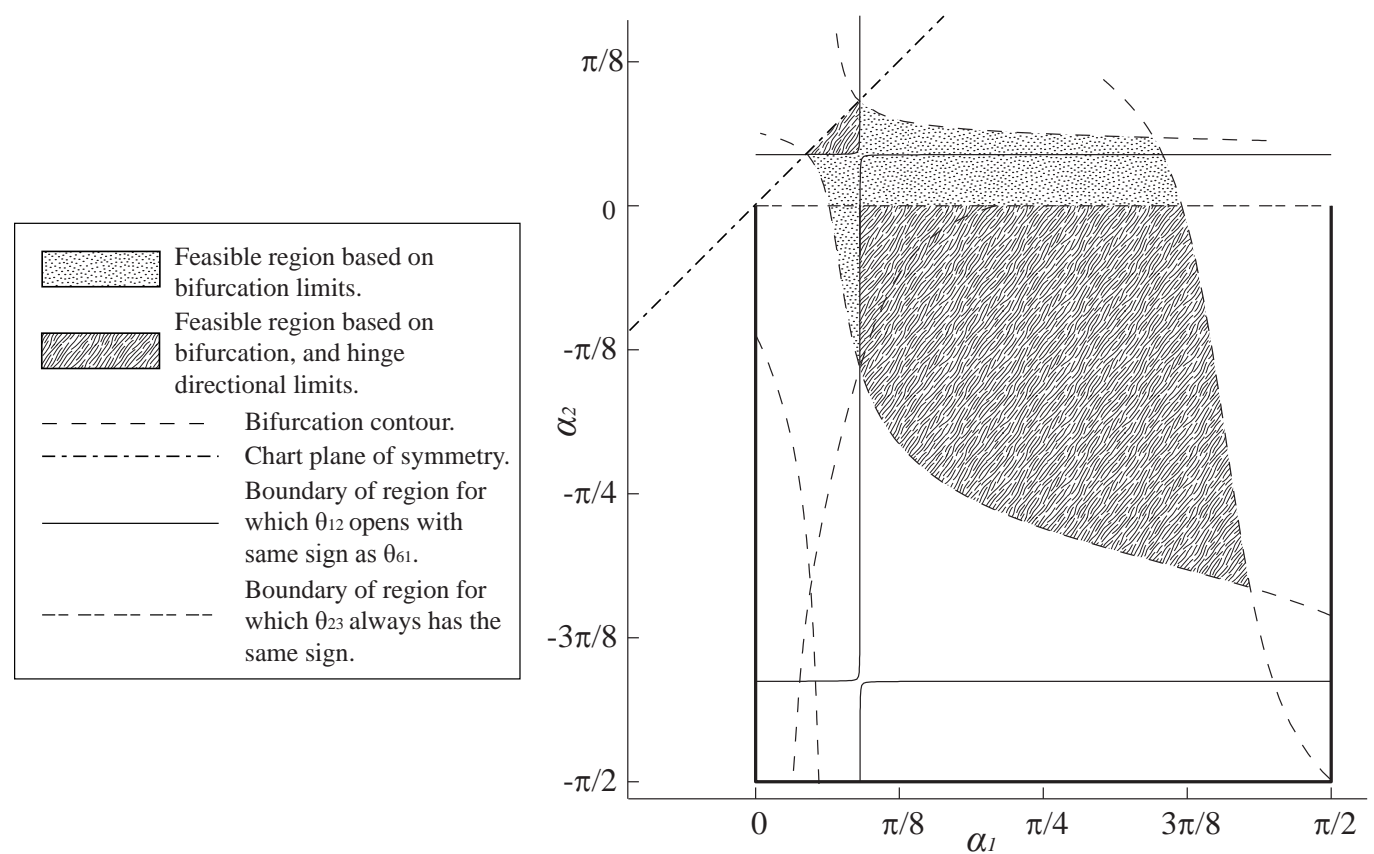

Figure 7: Feasibility map for $\gamma=5 \pi / 8$.

Points of note about these feasibility graphs are:

- we choose the range of values for $\alpha_{1}$ and $\alpha_{2}$ as $(-\pi / 2, \pi / 2]$ (other values can be mapped into this range);

- we choose the range of values for $\gamma$ as $(-\pi, \pi]$ (other values can be mapped into this range);

- there is always a plane of symmetry defined by $\alpha_{1}=\alpha_{2}$;

- plots for the range $\gamma \in(-\pi, 0]$ can be obtained by using $\gamma=\gamma_{0}-\pi$, where $\gamma_{0}$ is in the range $(0, \pi]$.

The loop closure equations (Equation 9) were used as the basis of construction for each of the contour lines in Figure 7. Many combinations of design variables will lead to a linkage which bifurcates somewhere in its range of movement. These combinations are marked as regular dashed lines. Some of the dashed lines represent bifurcations which occur outside the standard stowed-deployed range of motion (often a configuration the linkage could only reach if it passed through itself), but they are included for completeness. The bifurcation contours of key interest are those which mark a boundary between linkage designs which move continuously from stowed to deployed, and those which do not. If the combination of variables which produce a linkage which bifurcates right at the perimeter of this range 


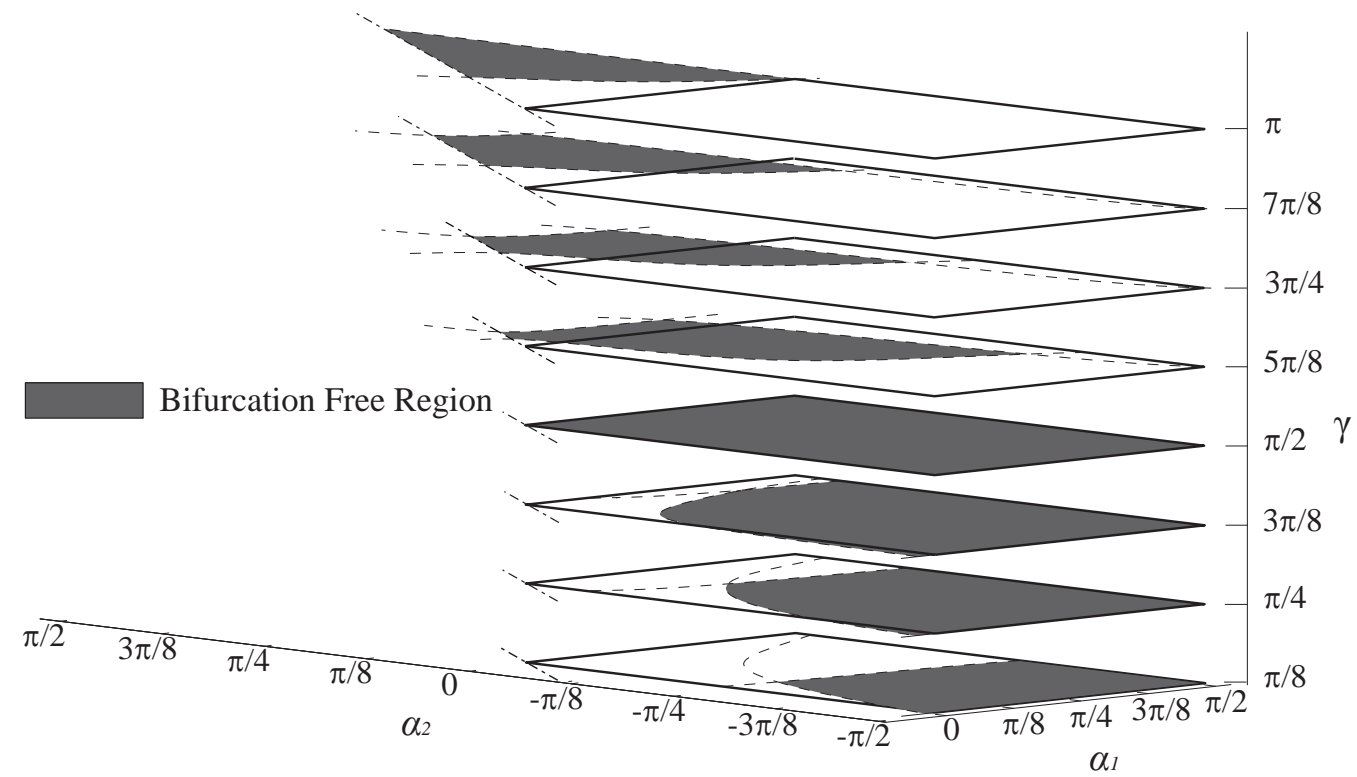

Figure 8: Variance of bifurcation free region with $\gamma$.

can be determined, then a region of design variable space which produces feasible linkages can be bounded. Because of the highly singular nature of the loop closure equations at a bifurcation point, standard predictor-corrector methods were not suitable for following the bifurcation paths through the design parameter space. Instead, a method which involved a (variable size) predictor step based on the previous step, and a subsequent unconstrained minimisation was used. The objective function has the form:

$$
F=\sigma_{5}+|\mathbf{F}|
$$

where $\sigma_{5}$ is the fifth singular value of the Jacobian of the loop closure equations (zero at a bifurcation), and $|\mathbf{F}|$ is the norm of the closure equations themselves (Equation 9). Note that it is also possible to find the linkage's singular values by constructing:

$$
D\left[\begin{array}{l}
\Delta \theta_{12} \\
\Delta \theta_{23} \\
\Delta \theta_{34} \\
\Delta \theta_{45} \\
\Delta \theta_{56} \\
\Delta \theta_{61}
\end{array}\right]=\left[\begin{array}{c}
\Delta x \\
\Delta y \\
\Delta z \\
\Delta \theta_{x} \\
\Delta \theta_{y} \\
\Delta \theta_{z}
\end{array}\right]
$$

where

$$
D=\left[\begin{array}{cccccc}
\mathbf{p}_{1} \times \mathbf{h}_{1} & \mathbf{p}_{2} \times \mathbf{h}_{2} & \mathbf{p}_{3} \times \mathbf{h}_{3} & \mathbf{p}_{4} \times \mathbf{h}_{4} & \mathbf{p}_{5} \times \mathbf{h}_{5} & \mathbf{p}_{6} \times \mathbf{h}_{6} \\
\mathbf{h}_{1} & \mathbf{h}_{2} & \mathbf{h}_{3} & \mathbf{h}_{4} & \mathbf{h}_{5} & \mathbf{h}_{6}
\end{array}\right]
$$

The singular values of $D$ can be used in just the same way as those of the Jacobian of Equation 9 .

The other contour lines, defining the boundaries of design for linkages with mono-directional hinges, were found using path followers which use a predictor step in the direction of the null space of the Jacobian at the previous point, and a corrector based simply on Newton's method.

For a larger collection of feasibility maps, see [24]. 


\section{Simulating the Linkage's Kinematics}

A loop closure equation based on each of the linkage's six hinge angles was derived in section 4. In this section, a similar equation is derived, but only in terms of two of the hinge angles. This equation will be referred to as a compatibility equation, as it ensures the compatibility of each half of the ring at the plane of symmetry. This is made possible by way of the assumption that the linkage is always symmetric about the plane defined by hinges $\mathbf{h}_{6}$ and $\mathbf{h}_{3}$. This assumption directly forms the basis of the compatibility equation, which can be written as:

$$
\Phi=\left[\left(\mathbf{p}_{6}-\mathbf{p}_{3}\right) \times \mathbf{h}_{6}\right] \cdot \mathbf{h}_{3}=0 .
$$

This equation can be written entirely in terms of the variable hinge angles $\theta_{61}$ and $\theta_{12}$ (as well as the fixed design parameters $\alpha_{1}, \alpha_{2}$ and $\gamma$ ). If one of the hinge angles, say $\theta_{61}$, is nominated as the driving, or input angle, then Equation 12 can be used to find $\theta_{12}$ in terms of $\theta_{61}$; the other four hinge angles can then be found.

If the locations of hinges 1 and $2\left(\mathbf{p}_{1}\right.$ and $\mathbf{p}_{2}$ ) are held fixed in space, then the locations of hinges 6 and 3 ( $\mathbf{p}_{6}$ and $\mathbf{p}_{3}$ ) can be found by rotating their locations in the deployed configuration about unit hinge vectors $\hat{\mathbf{h}}_{1}$ and $\hat{\mathbf{h}}_{2}$. To rotate a vector $\mathbf{v}$ about a (unit) axis $\mathbf{w}$ by an angle $\theta$ :

$$
\mathbf{v}^{\prime}=(\mathbf{v} \cdot \mathbf{w}) \mathbf{w}+(\mathbf{v}-(\mathbf{v} \cdot \mathbf{w}) \mathbf{w}) \cos \theta+\mathbf{v} \times \mathbf{w} \sin \theta
$$

If the axis $\mathbf{w}$ passes through a point $\mathbf{p}$, and the substitution $\mathbf{u}=\mathbf{v}-\mathbf{p}$ is made, then:

$$
\mathbf{v}^{\prime}=(\mathbf{u} \cdot \mathbf{w}) \mathbf{w}+(\mathbf{u}-(\mathbf{u} \cdot \mathbf{w}) \mathbf{w}) \cos \theta+\mathbf{u} \times \mathbf{w} \sin \theta+\mathbf{p}
$$

Applying Equation 14 to the current problem for $\mathbf{p}_{1}$ and $\mathbf{p}_{2}$ gives:

$$
\begin{aligned}
& \mathbf{p}_{6}=\left(\mathbf{u}_{1} \cdot \hat{\mathbf{h}}_{1}\right) \hat{\mathbf{h}}_{1}+\left(\mathbf{u}_{1}-\left(\mathbf{u}_{1} \cdot \hat{\mathbf{h}}_{1}\right) \hat{\mathbf{h}}_{1}\right) \cos \theta_{61}+\mathbf{u}_{1} \times \hat{\mathbf{h}}_{1} \sin \theta_{61}+\mathbf{p}_{1}(0) \\
& \mathbf{p}_{3}=\left(\mathbf{u}_{2} \cdot \hat{\mathbf{h}}_{2}\right) \hat{\mathbf{h}}_{2}+\left(\mathbf{u}_{2}-\left(\mathbf{u}_{2} \cdot \hat{\mathbf{h}}_{2}\right) \hat{\mathbf{h}}_{2}\right) \cos \theta_{12}+\mathbf{u}_{2} \times \hat{\mathbf{h}}_{2} \sin \theta_{12}+\mathbf{p}_{2}(0)
\end{aligned}
$$

where $\mathbf{u}_{1}=\mathbf{p}_{6}(0)-\mathbf{p}_{1}(0)$ and $\mathbf{u}_{2}=\mathbf{p}_{3}(0)-\mathbf{p}_{2}(0)$. Hinge vectors $\mathbf{h}_{6}$ and $\mathbf{h}_{3}$ also change during the folding/unfolding process. Applying Equation 13 to the problem of finding the hinge orientations gives:

$$
\begin{aligned}
& \hat{\mathbf{h}}_{6}=\left(\hat{\mathbf{h}}_{6}(0) \cdot \hat{\mathbf{h}}_{1}\right) \hat{\mathbf{h}}_{1}+\left(\hat{\mathbf{h}}_{6}(0)-\left(\hat{\mathbf{h}}_{6}(0) \cdot \hat{\mathbf{h}}_{1}\right) \hat{\mathbf{h}}_{1}\right) \cos \theta_{61}+\hat{\mathbf{h}}_{6}(0) \times \hat{\mathbf{h}}_{1} \sin \theta_{61} \\
& \hat{\mathbf{h}}_{3}=\left(\hat{\mathbf{h}}_{3}(0) \cdot \hat{\mathbf{h}}_{2}\right) \hat{\mathbf{h}}_{2}+\left(\hat{\mathbf{h}}_{3}(0)-\left(\hat{\mathbf{h}}_{3}(0) \cdot \hat{\mathbf{h}}_{2}\right) \hat{\mathbf{h}}_{2}\right) \cos \theta_{12}+\hat{\mathbf{h}}_{3}(0) \times \hat{\mathbf{h}}_{2} \sin \theta_{12}
\end{aligned}
$$

Assume that the bar connecting hinges 1 and 2 is fixed in space (i.e., the plane of symmetry moves). Since the position of hinge 6 is being rotated about $\hat{\mathbf{h}}_{1}$, it is possible to use Equation 16 to decompose $\mathbf{h}_{6}$ using:

$$
\begin{aligned}
& \mathbf{a}_{6}=\left(\hat{\mathbf{h}}_{6}(0) \cdot \hat{\mathbf{h}}_{1}\right) \hat{\mathbf{h}}_{1} \\
& \mathbf{b}_{6}=\left(\hat{\mathbf{h}}_{6}(0)-\left(\hat{\mathbf{h}}_{6}(0) \cdot \hat{\mathbf{h}}_{1}\right) \hat{\mathbf{h}}_{1}\right) \\
& \mathbf{c}_{6}=\hat{\mathbf{h}}_{6}(0) \times \hat{\mathbf{h}}_{1}
\end{aligned}
$$

and thus

$$
\begin{aligned}
& \hat{\mathbf{h}}_{6}=\mathbf{a}_{6}+\mathbf{b}_{6} \cos \theta_{61}+\mathbf{c}_{6} \sin \theta_{61} \\
& \hat{\mathbf{h}}_{3}=\mathbf{a}_{3}+\mathbf{b}_{3} \cos \theta_{12}+\mathbf{c}_{3} \sin \theta_{12}
\end{aligned}
$$

The positions of the hinges can also be parameterised as:

$$
\begin{aligned}
& \mathbf{q}_{6}=\left(\mathbf{u}_{1} \cdot \hat{\mathbf{h}}_{1}\right) \hat{\mathbf{h}}_{1}+\mathbf{p}_{1}(0) \\
& \mathbf{r}_{6}=\left(\mathbf{u}_{1}-\left(\mathbf{u}_{1} \cdot \hat{\mathbf{h}}_{1}\right) \hat{\mathbf{h}}_{1}\right) \\
& \mathbf{s}_{6}=\mathbf{u}_{1} \times \hat{\mathbf{h}}_{1}
\end{aligned}
$$

leading to:

$$
\begin{aligned}
& \mathbf{p}_{6}=\mathbf{q}_{6}+\mathbf{r}_{6} \cos \theta_{61}+\mathbf{s}_{6} \sin \theta_{61} \\
& \mathbf{p}_{3}=\mathbf{q}_{3}+\mathbf{r}_{3} \cos \theta_{12}+\mathbf{s}_{3} \sin \theta_{12}
\end{aligned}
$$


Substituting these forms into Equation 12, and collecting trigonometric terms leads to:

$$
\eta_{0}+\eta_{1} \cos \theta_{12}+\eta_{2} \sin \theta_{12}+\eta_{3} \sin \theta_{12} \cos \theta_{12}+\eta_{4} \cos ^{2} \theta_{12}+\eta_{5} \sin ^{2} \theta_{12}=0
$$

where $\eta_{i}$ can be expressed explicitly in terms of $\theta_{61}$ as well as $\mathbf{a}_{6}, \mathbf{b}_{6} \ldots \mathbf{q}_{6}, \mathbf{r}_{6} \ldots$ The process can just as easily be reversed to write Equation 17 in terms of $\theta_{61}$ with $\mathbf{a}_{6}, \mathbf{b}_{6} \ldots \mathbf{q}_{6}, \mathbf{r}_{6} \ldots$ found by choosing a value of $\theta_{12}$. It can be shown that in fact:

$$
\begin{aligned}
& \eta_{3}=0 \\
& \eta_{4}=\eta_{5}
\end{aligned}
$$

If a new definition is made for the trigonometric terms:

$$
\sin \theta_{12}=\frac{l}{\sqrt{1+l^{2}}}
$$

then it can be shown that:

$$
l=\frac{\eta_{1} \eta_{2} \pm\left(\eta_{0}+\eta_{4}\right) \sqrt{\eta_{1}^{2}+\eta_{2}^{2}-\left(\eta_{0}+\eta_{4}\right)^{2}}}{\left(\eta_{0}-\eta_{2}+\eta_{4}\right)\left(\eta_{0}+\eta_{2}+\eta_{4}\right)}
$$

Equation 18 can be used to find the positions and orientations of hinges 6 and 3. Using this information, it is possible to reflect in the plane defined by hinges 6 and 3 to find the positions and orientations of hinges 5 and 4 . Hinge angles $\theta_{56}$ and $\theta_{23}$ can be found easily in terms of the angles between the plane of symmetry and the bars connecting hinges 1 and 6 , and 2 and 3 . An example of how the hinge angles $\theta_{61}$ and $\theta_{12}$ vary with respect to one-another during unfolding is given in Figure 9.

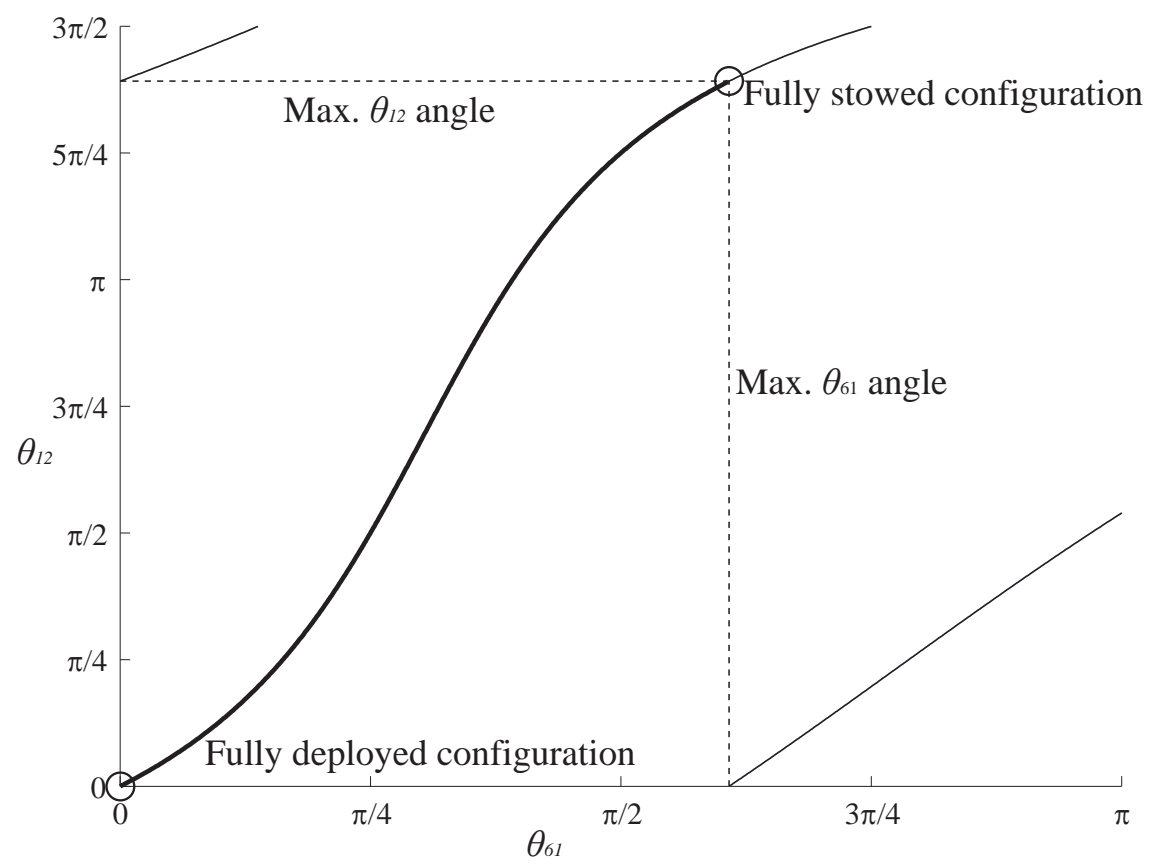

Figure 9: Angle evolution during the deployment of a linkage with parameters $\alpha_{1}=\pi / 4, \alpha_{2}=-\pi / 4$ and $\gamma=\pi / 2$, derived from Equation 18. 


\section{Using a Polyhedral Homotopy to Design Six-Bar Linkages}

Having now attained an expression for the compatibility equation in terms of $\alpha_{1}, \alpha_{2}$ and $\gamma$, Equation 12 can be written as a pure polynomial in terms of variables replacing the set of sines and cosines $\left\{C_{\alpha_{1}}, S_{\alpha_{1}}, C_{\alpha_{2}}, S_{\alpha_{2}}, C_{\gamma}, S_{\gamma}\right\}$. The polynomial itself is quite long, and in its fully expanded form consists of 389 distinct terms, prohibiting any manual manipulation, and making its explicit representation difficult. The equation can be formed as a black-box function, with inputs $\mathbf{x}=\left\{C_{\alpha_{1}}, S_{\alpha_{1}}, C_{\alpha_{2}}, S_{\alpha_{2}}, C_{\gamma}, S_{\gamma}\right\}$, and extra parameters $\left\{\theta_{61}, \theta_{12}, l\right\}$. The compatibility equation can be written as:

$$
\Phi\left(\mathbf{x}, \theta_{61}, \theta_{12}, l\right)=0
$$

If the function evaluates to zero, then the equation is satisfied and the inputs define a compatible linkage configuration.

The compatibility equation for the plane symmetric rectangular 6-bar linkage was derived in terms of three variables, $\alpha_{1}, \alpha_{2}$ and $\gamma$. All three are angles, and are considered to be the only design variables for the linkage for the purposes of this section.

Due to Equation 1, it is implicit that the linkage will start as a rectangular structure with length twice its width, and then fold into a compact configuration in which all the bars are parallel. This is arguably the most desirable characteristic of the linkage should one intend to use it as a deployable structure. It is possible, however, that one might like to control the way in which the linkage opens and folds. This might be desirable in order to minimise the stretch on a flexible sheet attached to the linkage, or perhaps to confine the dimensions of the linkage to a particular three dimensional envelope during its opening. The complexity of the equations involved in describing the motion of the linkage means that only numerical optimisation techniques lend themselves to any attempt to modify the linkage's parameters of motion. It is also possible, however, to 'guide' the linkage on its way from deployed to folded and vice versa by defining intermediate configurations through which is must pass. Since the linkage has only a single degree of freedom, the specification of the angle between any two adjacent bars is sufficient to completely describe the state of the linkage. It is not possible, in general, to specify a continuous relationship between any two hinge angles as this over-determines the system. It is possible to specify the values of more than one hinge angle in the linkage during the opening and closing process at a discrete number of positions. For each of these discrete positions, one of the design variables must be freed up in order to keep the system determined. Since there are only three design variables, a maximum of three discrete positions may be specified on the linkage's path.

An appropriate way to specify positions along the opening and closing path is to use the hinge angles $\theta_{61}$ and $\theta_{12}$ in matched pairs $\left\{\theta_{61}, \theta_{12 j}\right\}$ for $j=1,2,3$ to establish a set of angular waypoints (or precision points) through which the linkage must pass. Note that it is not possible to specify the order in which the waypoints are encountered during an opening/closing run. It is also not possible to determine whether the linkage will self-intersect during opening/closing. This can only be investigated using simulation.

In choosing to consider the sines and cosines of the variables instead of the variables themselves, it has become necessary to introduce a set of equations to compensate for the increase in the number of polynomial variables, as was done in Equation 11. This can be achieved in a number of ways, but a simple way is given in Equation 19, preserving polynomial form.

$$
\begin{aligned}
C_{\alpha_{1}}^{2}+S_{\alpha_{1}}^{2}-1 & =0 \\
C_{\alpha_{2}}^{2}+S_{\alpha_{2}}^{2}-1 & =0 \\
C_{\gamma}^{2}+S_{\gamma}^{2}-1 & =0
\end{aligned}
$$

Design problems can now be solved using the compatibility equation. The particular type of problem considered here involves using pre-specified parameter sets $\left\{\theta_{61_{i}}, \theta_{12_{i}}\right\}$ and then solving for a $\left\{C_{\alpha_{1}}, S_{\alpha_{1}}, C_{\alpha_{2}}, S_{\alpha_{2}}, C_{\gamma}, S_{\gamma}\right\}$ set which satisfies the equations. The full equation set is given in Equation 20.

$$
F=\left\{\begin{array}{l}
\Phi\left(\mathbf{x}, \theta_{611}, \theta_{121}, l\right) \\
\Phi\left(\mathbf{x}, \theta_{612}, \theta_{122}, l\right) \\
\Phi\left(\mathbf{x}, \theta_{613}, \theta_{123}, l\right) \\
C_{\alpha_{1}}^{2}+S_{\alpha_{1}}^{2}-1 \\
C_{\alpha_{2}}^{2}+S_{\alpha_{2}}^{2}-1 \\
C_{\gamma}^{2}+S_{\gamma}^{2}-1
\end{array}\right\}=\mathbf{0}
$$


Equation 20 is all that is required to form a polyhedral homotopy of the type described [22], p.138. It is a system of polynomial equations in six variables. Each of the first three equations of 20 contains the same set of 389 monomials, which means they have an identical support (polynomial structure) with 389 elements. It is possible to use Mathematica [17] to analyse the full equations and arrange the supports into matrix form. The mixed volume of the supports is 2352 , meaning that the system has at most this many solutions. It is worth noting that if the variables are placed into homogeneous groups $\left[\left\{C_{\alpha_{1}}, S_{\alpha_{1}}\right\},\left\{C_{\alpha_{2}}, S_{\alpha_{2}}\right\},\left\{C_{\gamma}, S_{\gamma}\right\}\right]$, the system has a Bézout number [25] of 2400. The proximity of the Bézout number and the mixed volume is due to the breadth of monomials present in the first three equations of the target system. It was found that the polyhedral homotopy method exhibited greater numerical stability than those using multi-homogenisation in this case, and hence this is the method used here.

Since the system of equations in 20 contains more than one equation with the same structure; that is, equations with the same polynomial structure but different coefficients, it is possible to use special polyhedral methods to simplify the process of constructing a start system for solving the target problem. The system is said to have a semi-mixed support. The first three equations, representing the compatibility equation but with different coefficients, is treated as a single equation, but with multiplicity three. The convex hull of the support of the first three equations $\left(Q_{1}\right)$ contains only 102 elements; a significant reduction on 389. It is these 102 which are dealt with directly when forming the polyhedral homotopy. In the notation of [26], the target equations of 20 have $n=6$ with $r=4: k_{1}=3, k_{2}=1, k_{3}=1, k_{4}=1$. That is to say, the system is written in terms of six unknowns, and there are six equations, but the first three have the same polynomial structure, leaving four distinct polynomial types. Also, $\operatorname{dim}\left(Q_{1}\right)=m_{1}=102, \operatorname{dim}\left(Q_{2}\right)=m_{2}=3$, $\operatorname{dim}\left(Q_{3}\right)=m_{3}=3$ and $\operatorname{dim}\left(Q_{4}\right)=m_{4}=3$.

Using continuation to follow the 2352 start solutions from the binomial start system to the random coefficient version of the target system, results in a full complement of non-singular finite solutions to track to the real coefficient system. This second continuation process, in which the solutions to the random complex coefficient system are tracked to those of the real coefficient target system, leaves only 500 non-singular finite solutions. These are the solutions of key interest.

\section{Examples of Solution Runs}

Some examples for essentially randomly chosen waypoints are given in Table 1. In each of the continuation runs, the number of non-singular finite solutions was in the region of 500. What is of interest is how many of those solutions are real. The number of real solutions is given, along with the number of these which were found to be geometrically meaningful and distinct.

In the first example, only one solution progresses smoothly from deployed to stowed (this is common), and in this case it has the design parameters:

$$
\begin{aligned}
\alpha_{1} & =-\pi / 4 \\
\alpha_{2} & =\pi / 4 \\
\gamma & =-\pi / 2
\end{aligned}
$$

This result is not particularly surprising as the waypoints were taken from a simulation of a linkage with these design variables. The second and third examples show similar results, with the number of potential solutions found varying. The results for the waypoints specified in Table 1, Example \# 4 show no practically desirable solutions. This example differs from the others in that the search was for a set of hinge angles not monotonically increasing in $\theta_{12}$ with respect to $\theta_{61}$. It is possible that no smooth solutions can be found for such a case.

It is important to remember that the theory of polynomial continuation guarantees that all solutions satisfying the waypoint constraints will be found. The solution sets given here for these particular examples can be said with confidence to be complete. 


\section{Summary of Triple Waypoint Examples}
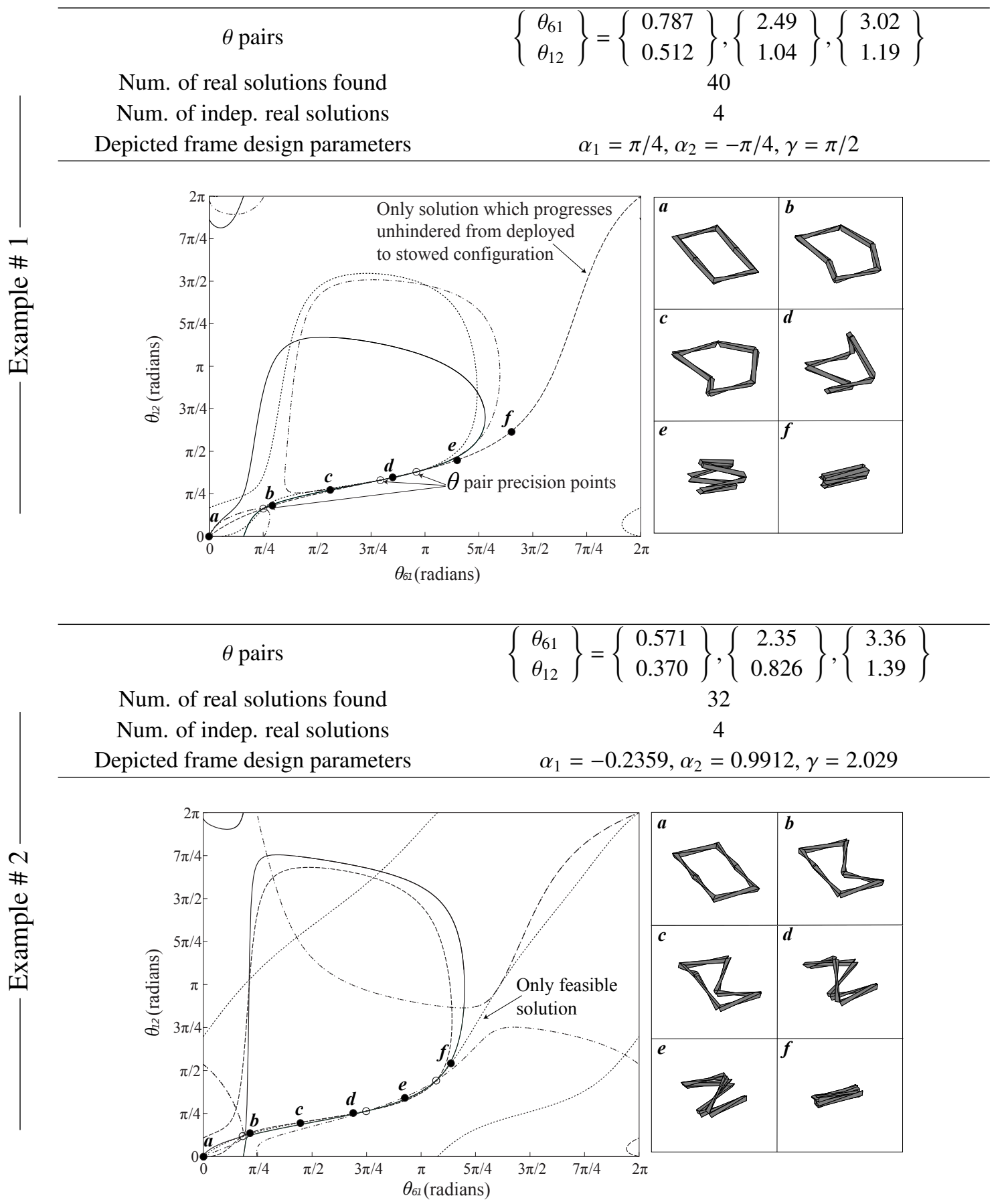

...Continued on next page 


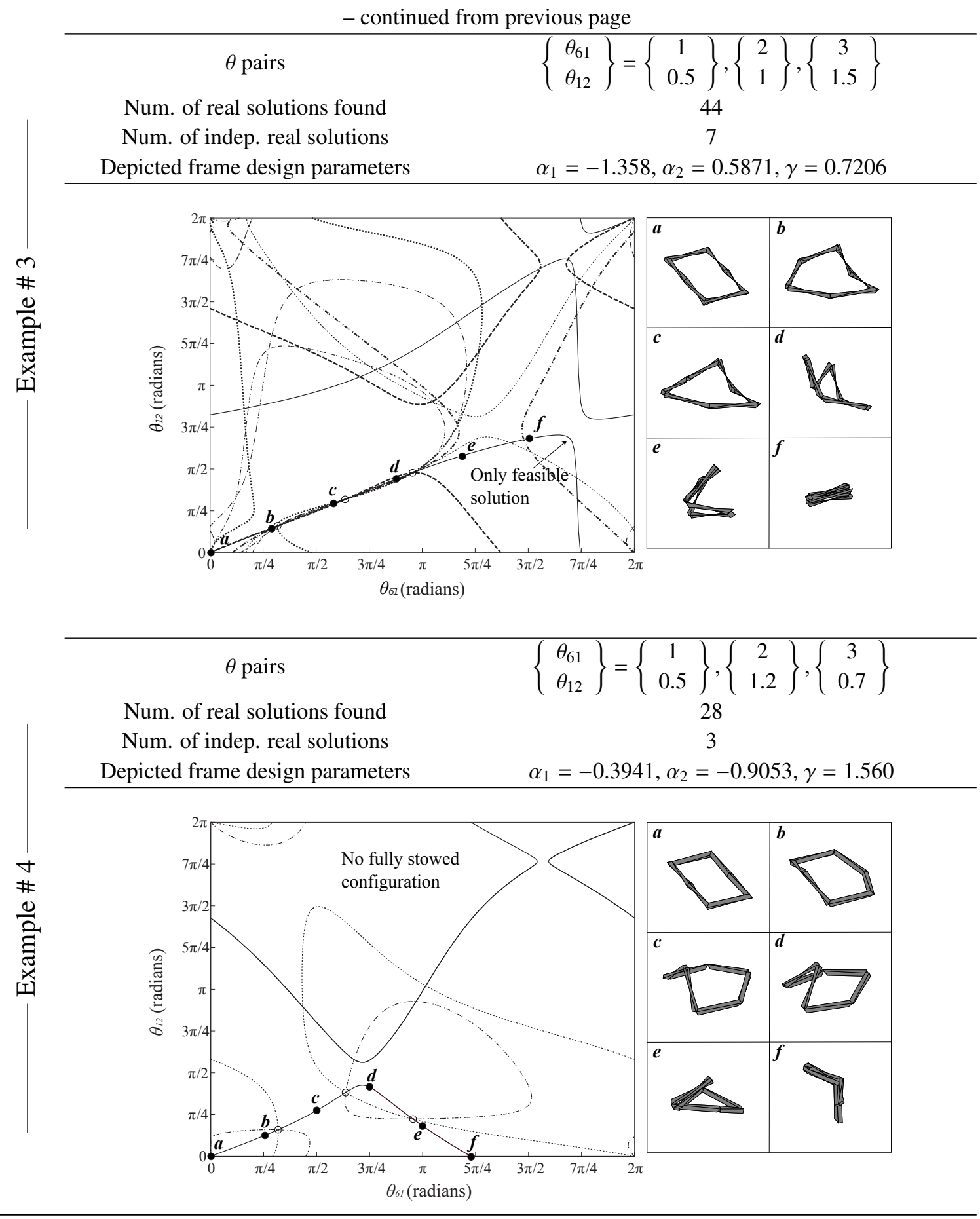

Table 1: Angle paths for triple waypoint examples. Each curve in each example represents a different linkage design. The $\theta$ pair waypoints are marked with circles. Note that the combination of $\theta_{61}$ and $\theta_{12}$ for which a linkage is said to be stowed will be different for each design. One particular design from each example has been chosen and represented at a variety of positions during the folding process, and its design parameters $\left(\alpha_{1}, \alpha_{2}\right.$ and $\left.\gamma\right)$ listed (the other designs' parameters have been omitted for brevity). The corresponding curve has been marked at the various deployment stages using solid circles. 


\section{Conclusion}

The mobility of the plane symmetric $6 \mathrm{R}$ foldable ring over-constrained mechanism has been shown to manifest itself in the linkage's closure equations as a single irreducible component in the one-dimensional solution set. A set of 'feasibility maps' showing the regions in parameter space in which the $6 \mathrm{R}$ foldable ring exhibits desirable characteristics has been produced. Also, a method of designing such rings by specifying angular waypoints has been demonstrated. It is hoped that these techniques, together, will provide a useful and practical way of designing plane symmetric, $6 \mathrm{R}$ foldable rings.

\section{References}

[1] R. Bricard, Mémoire sur la théorie de l'octaèdre articulé, Journal de mathématiques pures et appliquées 3 (1897) 113-148.

[2] R. F. Crawford, J. M. Hedgepeth, P. R. Preiswerk, Spoked wheels to deploy large surfaces in space-weight estimates for solar arrays, Tech. Rep. NASA-CR-2347; ARC-R-1004, NASA (1975)

[3] Y. Chen, Z. You, T. Tarnai, Threefold-symmetric Bricard linkages for deployable structures, International Journal of Solids and Structures 42 (2004) 2287-2301.

[4] Y. Chen, Z. You, Square deployable frames for space applications. part 1: theory, Journal of Aerospace Engineering 220 (2006) $347-354$.

[5] Y. Chen, Z. You, Square deployable frames for space applications. part 2: realization, Journal of Aerospace Engineering 221 (2006) 37-45.

[6] Y. Chen, Design of structural mechanisms, Ph.D. thesis, University of Oxford (2003).

[7] W. W. Gan, S. Pellegrino, Closed-loop deployable structures, in: 44th AIAA/ASME/ASCE/AHS Structures, Structural Dynamics, and Materials Conference, 2003

[8] K. Wohlhart, A new 6R space mechanism, in: Proceedings of the 7th World.Congress on the Theory of Machines and Mechanisms, Vol. 1, Sevilla, Spain, 1987., pp. 193-198.

[9] K. Wohlhart, The two types of the orthogonal Bricard linkage, Mechanism and Machine Theory 28 (1993) $809-817$.

[10] L. Racila, M. Dahan, Spatial properties of Wohlhart symmetric mechanism, Meccanica 45 (2010) 153-165.

[11] S. Pellegrino, C. Green, S. D. Guest, A. Watt, SAR advanced deployable structure, Tech. rep., University of Cambridge Department of Engineering (2000).

[12] W. W. Gan, S. Pellegrino, Kinematic bifurcations of closed-loop deployable frames, in: Proceedings of the 5th International Conference on Computation of Shell and Spatial Structures, 2005.

[13] W. W. Gan, S. Pellegrino, Numerical approach to the kinematic analysis of deployable structures forming a closed loop, Journal of Mechanical Engineering Science 220 (C) (2006) 1045-1056.

[14] Y. Chen, Z. You, Two-fold symmetrical 6R foldable frame and its bifurcations, International Journal of Solids and Structures 46 (2009) $4504-4514$.

[15] A. J. Sommese, J. Verschelde, Numerical homotopies to compute generic points on positive dimensional algebraic sets, Journal of Complexity 16 (2000) 572-602.

[16] J. Baker, An analysis of the Bricard linkages, Mechanism and Machine Theory 15 (1980) 267-286.

[17] Mathematica, Version 7.0, Wolfram Research, Inc., Champaign, Illinois, 2008.

[18] F. Freudenstein, E. R. Maki, The creation of mechanisms according to kinematic structure and function, Environment and Planning B 6 (1979) 375-391.

[19] A. J. Sommese, J. Verschelde, C. W. Wampler, Numerical decomposition of the solution sets of polynomial systems into irreducible components, SIAM Journal of Numerical Analysis 38 (6) (2001) 2022-2046.

[20] E. L. Allgower, K. Georg, Introduction to Numerical Continuation Methods, Society for Industrial and Applied Mathematics (SIAM), 2003.

[21] A. Morgan, Solving Polynomial Systems Using Continuation for Engineering and Scientific Problems, Prentice-Hall, inc., 1987.

[22] A. J. Sommese, C. W. Wampler, The Numerical Solution of Systems of Polynomials Arising in Engineering and Science, World Scientific, 2005.

[23] T. Gao, T.-Y. Li, Mixed volume computation for semi-mixed systems, Discrete and Computational Geometry 29 (2) (2003) $257-277$.

[24] A. Viquerat, Polynomial continuation in the design of deployable structures, Ph.D. thesis, University of Cambridge (2011).

[25] C. W. Wampler, A. P. Morgan, A. J. Sommese, Numerical continuation methods for solving polynomial systems arising in kinematics, Journal of Mechanical Design 112 (1990) 59-68.

[26] T. Y. Li, Handbook of Numerical Analysis, Vol. XI, North-Holland, 2003, Ch. Numerical Solution of Polynomial Systems by Homotopy Continuation Methods, pp. 209-304. 